Ciencia y Educación, Vol. 4, No. 3, septiembre-diciembre, 2020

ISSN (impreso): 2613-8794 • ISSN (en línea): 2613-8808

DOI: https://doi.org/10.22206/cyed.2020.v4i3.pp27-43

\title{
Enseńanza de Historia en secundaria: experiencia en investigación-acción en República Dominicana
}

\author{
Teaching of History in high school: action research \\ experience in Dominican Republic
}

\author{
Anny Vásquez ${ }^{a}$ ORCID: 0000-0002-5565-9684 \\ Albreydy Holguin ${ }^{\text {b }}$ ORCID: 0000-0002-0152-7299
}

Recibido: 28/02/2020 • Aprobado: 14/05/2020

\begin{abstract}
Cómo citar: Vásquez, A., \& Holguin, A. (2020). Enseñanza de Historia en secundaria: experiencia en investigación-acción en República Dominicana. Ciencia y Educación, 4(3), 27-43. Doi: https://doi.org/10.22206/cyed.2020.v4i3.pp27-43
\end{abstract}

\section{Resumen}

El presente artículo relata una experiencia de investigación-acción sobre estrategias lúdicas para la enseñanza de Historia en el nivel secundario. En la misma participaron 25 estudiantes, de edades entre los 12 y 14 años, dos docentes y una coordinadora pedagógica del 2do grado de secundaria de un centro educativo privado del Distrito Nacional en República Dominicana. El estudio se desarrolló con un carácter cualitativo y descriptivo, a través de observaciones, entrevistas, grupos focales y pruebas diagnósticas. Realizada la intervención, se concluye que los estudiantes aprenden Historia de forma significativa cuando se utilizan estrategias que fomentan su participación, favoreciendo así el desarrollo de competencias, lo que supone a los docentes un motivo de reflexión sobre el acercamiento de su práctica al desarrollo del aprendizaje significativo y el posible diseño de planes de mejora respecto a la enseñanza de la Historia.

Palabras claves: estrategias de enseñanza; juego educativo; enseñanza de Historia; educación secundaria; investigación acción.

\begin{abstract}
This study reports an action - research experience on playful strategies for teaching history in high school. This project counted with the participation of two teachers and 25 students, aged between 12 and 14 years old, of the 2 nd grade of a private school in the Distrito Nacional of the Dominican Republic. The research method was qualitative descriptive, based on data collected through interviews, focus groups, participant observation, and diagnostic tests. Having performed the intervention, it is concluded that students learn history in a meaningful way when strategies foster their participation, favoring the development of their capacities and abilities, which represents for teachers a motive for reflection on the approach of their practice towards meaningful learning and the need for improvement plans regarding the teaching of history.
\end{abstract}

Keywords: Teaching methods; educational games; History education; secondary education; action research.

\footnotetext{
a Instituto Tecnológico de Santo Domingo (INTEC), República Dominicana Correo-e: annyclarisa15@gmail.com,1073459@est.intec.edu.do

a Instituto Tecnológico de Santo Domingo (INTEC), República Dominicana

Correo-e: albreydy@gmail.com,1073063@est.intec.edu.do
} 


\section{Introducción}

Alrededor del mundo, el aprendizaje de historia universal y local es concebido como imprescindible en los programas educativos. El análisis de los fenómenos y procesos históricos incide en el desarrollo del pensamiento crítico, así como estimula la comprensión de las sociedades contemporáneas (Prats, 2007). La enseñanza de la historia debe estar orientada a que el estudiantado desarrolle una comprensión más profunda y crítica de su entorno. Sus experiencias se enriquecen mientras estudian épocas y sociedades distintas a las que pertenecen, al mismo tiempo que reflexionan críticamente sobre lo que aprenden (Pluckrose, 1996). Sin embargo, en República Dominicana la enseńanza de Historia en secundaria se aleja del desarrollo del aprendizaje significativo, presentando profundas deficiencias. De acuerdo con los Informes Curriculares de las Pruebas Nacionales aplicadas por el Ministerio de Educación de la República (MINERD), en los últimos cuatro años el estudiantado, tanto en su modalidad general como en técnico-profesional, presenta los porcentajes más bajos de dominio en los bloques de contenidos de Historia respecto a los demás bloques del área de Ciencias Sociales (MINERD, 2016; 2017; 2018). Estos exámenes son pruebas estandarizadas que se aplican a final de cada año escolar a la población estudiantil del último grado de primaria y secundaria, para evaluar los aprendizajes obtenidos a lo largo de cada uno de estos niveles, y como validación para la promoción hacia la educación superior.

Según Casal (2011), la enseñanza de Historia en secundaria se fundamenta tradicionalmente en memorizar las biografías o hazañas de personajes, fechas y hechos. Esta problemática surge y está acompañada de un conjunto de malas prácticas. Entre ellas, podemos citar las siguientes: a) la separación entre los contenidos y las realidades contemporáneas; b) la superposición de lo conceptual ante lo procedimental y actitudinal; c) el abuso del libro de texto y la cátedra magistral como estrategia didáctica; d) el escaso dominio del contenido disciplinar; e) la super- posición de los aspectos políticos ante los culturales, sociales y económicos; f) la falta de reflexión y crítica; y g) la poca relación entre los planteamientos curriculares y su ejecución por parte de los y las docentes (Reyes, Jevey, Guerra, Palomo y Romero, 2009).

El Diseño Curricular del Nivel Secundario, del Ministerio de Educación de la República Dominicana (MINERD, 2016) propone diferentes medios y recursos para el aprendizaje de Ciencias Sociales, partiendo de un enfoque de educación por competencias y de un paradigma histórico-cultural. Con el uso de estas estrategias y recursos, se espera que el estudiantado se ubique en el tiempo y el espacio, que interprete los hechos a partir de su contexto, que critique los cambios ocurridos en nuestro mundo, que compare fuentes de información, que reflexione sobre los avances y retrocesos de las naciones en los últimos dos siglos, que relacione el impacto de las ideas filosóficas en la sociedad, la política, la economía y el espacio, que discuta sobre las diferencias existentes entre países y, finalmente, que respete el medio ambiente a través de acciones y posiciones críticas. Sin embargo, el desarrollo de estas competencias se ve entorpecido por las prácticas deficientes, mencionadas anteriormente.

Sáiz y Fuster (2014) plantean que:

aprender historia no puede limitarse sólo a memorizar y repetir conocimientos académicos o narrativas del pasado. Ello equivale a confundir pasado histórico e historia como construcción narrativa de ese pasado. Aprender historia debe suponer también y, sobre todo, aprender a representar y significar ese pasado, a construir conocimientos históricos propios y utilizarlos para pensar y comprender la realidad social (p. 48).

El estudio realizado por Sosa y Rodríguez (2010), intitulado Métodos y Técnicas de la Enseñanza de la Historia y la Geografía en el Segundo Ciclo del Nivel Medio, en un liceo público de la provincia de Santiago de los Caballeros, de República Dominicana, buscó proponer prácticas para producir una 
mejora en las técnicas y métodos que los docentes del segundo ciclo del Nivel Medio utilizan para enseñar Historia y Geografía. Entre sus resultados se destaca la preferencia del cuerpo docente al uso de métodos de enseñanza tradicionales, y una escasez significativa en el uso de estrategias que impliquen al rol activo del estudiante.

Otra investigación (Casal, 2011), realizada en Michoacán, México, se basó en un análisis tanto cuantitativo como cualitativo para efectuar un diagnóstico del proceso de enseńanza-aprendizaje en tres escuelas secundarias generales, realizando un seguimiento al aprendizaje de los estudiantes. El estudio determinó que los estudiantes prefieren tener un rol activo frente al aprendizaje, ser creativos en vez de receptivos, y realizar juicios críticos, en lugar de factuales.

\section{Marco teórico}

\section{Estrategias de enseñanza-aprendizaje}

Las estrategias de enseñanza-aprendizaje son un engranaje de procedimientos que se seleccionan para lograr la adquisición, memorización y posterior utilización de la información (Hurtado, García, Rivera y Forgiony, 2018). Es decir, son secuencias de actividades integradas que comparten el objetivo de promover el aprendizaje.

Desde un enfoque por competencias, las estrategias de enseñanza buscan el fortalecimiento de las capacidades, los saberes y las destrezas de los estudiantes de manera eficiente (Cepeda, 2013). Estos postulados concuerdan con lo establecido por el MINERD en las Bases de la Revisión y Actualización Curricular (2016), donde se contemplan las estrategias de enseñanza-aprendizaje como la secuencia de actividades que el docente planifica y organiza de forma sistemática para favorecer y potenciar la construcción del saber mientras conducen al estudiante a enfrentar situaciones en las que aplique sus conocimientos. En ese orden, el MINERD sugiere las siguientes estrategias: estrategias de recuperación de experiencias previas, estrategias expositivas de conocimientos elaborados y/o acumulados, estrategias de descubrimiento e indagación de información, estrategias de inserción en el entorno, estrategias de indagación dialógica, aprendizaje basado en problemas, sociodrama o dramatización, estudios de casos y debates.

\section{El proceso de enseñanza-aprendizaje en historia}

El propósito principal, en cuanto al aprendizaje de la historia, es brindar a los estudiantes las herramientas necesarias para comprender el mundo en el que viven, las experiencias colectivas del pasado y la actualidad y el espacio en que estas se desarrollan, a la vez que identifican los procesos y mecanismos que rigen los hechos sociales; interrelacionan hechos políticos, económicos y culturales; comprenden la multiplicidad de causas que explican la evolución de la sociedad; localizan e identifican en el espacio y el tiempo los procesos históricos relevantes; desarrollan respeto por las diferencias, y buscan, distinguen, y comprenden información de naturaleza y fuentes diversas (Molina, Miralles, Deusdad y Begoña, 2017). Estas competencias se encuentran en armonía con las expectativas plasmadas en el Diseño Curricular del Nivel Secundario (MINERD, 2016).

En la actualidad, las sociedades han reducido la importancia de la historia a la justificación de acciones políticas, sociales y culturales del presente, lo que ha afectado la relevancia que tiene esta área del saber, (Prats y Santacana, 2001). En ese sentido, de acuerdo con Reyes et al. (2009), entre las prácticas deficientes en la enseñanza de historia se pueden destacar las siguientes: los contenidos son fraccionados y enseñados de forma lineal, olvidando el carácter dialéctico de la historia; se resta importancia a la parte procedimental y actitudinal del aprendizaje, priorizando lo conceptual; se privilegia el aprendizaje mecánico, basado en la memorización, sobre el aprendizaje significativo, el cual requiere de mayor dedicación y tiempo por parte del docente en la preparación de secuencias didácticas; falta de relación entre los 
contenidos disciplinares y la realidad social en la que se ubican los estudiantes; exceso de uso de estrategias expositivas; manejo del libro de texto como único recurso y prioridad de los aspectos políticos sobre los sociales, culturales y económicos, ignorando la interdisciplinariedad de las ciencias sociales, prácticas que guardan relación con lo planteado por Casal (2011).

Debido a estas y otras dificultades es que los estudiantes memorizan los contenidos impartidos en las clases de Historia sin desarrollar pensamiento histórico, como corroboran Sáiz y Fuster (2014). Esta problemática se genera en un contexto en donde el aprendizaje de la historia se ha concebido como la simple memorización de hechos y fechas. A razón de esto Casal (2011) y Sáiz y Fuster (2014) proponen integrar los conocimientos y las habilidades de comprensión, es decir, contenidos y competencias. Piensan que aprender historia debe suponer representar e identificar el pasado, construir conocimientos históricos propios y usarlos para pensar, comprender y actuar sobre la realidad social.

\section{Estrategias de enseñanza-aprendizaje lúdicas}

En el campo de la investigación educativa y de las neurociencias, se utilizan diferentes conceptos para abordar la relación entre la diversión y el aprendizaje. Benítez (2010) habla sobre el "juego didáctico", refiriéndose a toda actividad recreativa que busca desarrollar capacidades a través de la participación estudiantil. Postula que tanto en el juego como en el aprendizaje se presentan retos que superar y la práctica como vía para el desarrollo de habilidades. Por otra parte, Córdoba, Lara y García (2017) teorizan sobre las "experiencias lúdicas", categorizándolas como expresiones que atraviesan aspectos sociales, personales, interpersonales, psicológicos y pedagógicos, que pueden constituirse como un recurso de aprendizaje que fomenta la creatividad y autonomía de los estudiantes. Desde la neuroeducación se plantea que el juego es un proceso inherente a todos los seres humanos; por cuanto segregamos neurotransmisores al participar de él. La dopamina, la serotonina, la oxitocina y las endorfinas son las sustancias respon- sables de la motivación, la generosidad, la regulación del estado anímico, y la felicidad, y todas guardan una estrecha relación con lo lúdico (Guillén, 2017).

Otros autores acogen también el término "lúdica", definiéndolo como un espacio recreativo que desarrolla habilidades intelectuales y socioafectivas mediante la alegría y el placer (Araujo, Gómez, Fonseca y Molano, 2013). Tomando estas referencias en cuenta, se apunta a construir entonces el concepto "estrategias de enseńanza-aprendizaje lúdicas" para hacer referencia a aquellas actividades que combinan la diversión y/o el placer con el aprendizaje y el desarrollo de destrezas. Son situaciones de aprendizaje integradas al contexto educativo de forma ingeniosa para fomentar la resolución de problemas y promover el desarrollo de competencias (Martínez y Pérez, 2015).

\section{Teoria del aprendizaje significativo}

La teoría del aprendizaje significativo fue postulada por David Ausubel en 1963 y reafirmada en el 2000, pero sigue vigente. Aunque en su momento Ausubel quizá asoció su planteamiento a las convencionales clases expositivas, ha podido mantenerse con las diferentes propuestas de enseñanza que se han desarrollado, como las clases a través de plataformas virtuales, libros, juegos, prácticas de laboratorio, entre otras estrategias y recursos. La idea fundamental ha sido que el estudiante no requiere del descubrimiento para lograr el aprendizaje, sino que puede asociar lo que sabe y lo que intenta aprender (Moreira, 2017).

Vallori, Enrique, Aguiló y Xamena (2002) plantean brevemente otros beneficios del aprendizaje significativo:

Conseguir que el alumnado tenga estructuras de conocimiento potentes y significativas hace que se sienta bien y que mejore su autoestima, que se sienta interesado por lo que aprende y que le guste lo que hace; tiene un fuerte estímulo intelectual, porque ve el resultado positivo de su proceso de aprendizaje, mantiene alta la moral del grupo y aprende a aprender (p. 101). 


\section{Metodología}

El presente estudio fue realizado bajo un enfoque cualitativo con alcance descriptivo, diseño que se caracteriza por estar orientado a la compresión de un fenómeno social a partir de sus determinantes (Guerrero, 2016). El diseño del estudio corresponde a la metodología de investigación-acción, a través de la cual las personas investigadas se convierten en participantes principales, construyéndose así el conocimiento a partir de su propia realidad, identificando dificultades y elaborando planes de acción orientados a resolver sus necesidades. A su vez, esta metodología se suscribe a un paradigma sociocrítico, que sostiene que las soluciones efectuadas suceden gracias a la crítica social y la reflexión de cada participante, de lo individual a lo colectivo y viceversa (Orozco, 2006).

El trabajo se realizó en un centro educativo privado ubicado en una zona comercial del Distrito Nacional, cuyo estudiantado pertenece a la clase económica alta y media alta. Participaron un total de 25 estudiantes, de edades entre los 12 y 14 años: 15 alumnas y 10 alumnos, del segundo grado de nivel secundario. También se contó con la participación de dos docentes de la asignatura de Ciencias Sociales y de la coordinadora pedagógica encargada del segundo grado, quienes dieron seguimiento al proceso. La técnica de muestreo fue no probabilística por conveniencia. De acuerdo con Otzen y Manterola (2017), esta técnica permite seleccionar individuos de una población que sean accesibles y aceptan ser incluidos. Es necesario destacar que el problema a investigar se determinó tras haber realizado cuatro semanas de observación participante en dicho centro educativo.

El diseño metodológico de este estudio se llevó a cabo a través de las fases en espiral del modelo de Kurt Lewin (1992) y se caracterizó por la necesidad de transformar una realidad (Pérez-Van-Leenden, 2019). De acuerdo con el esquema de Carr y Kemmis (citado en Abero, Berardi, Capocasale, García y Rojas, 2015), las fases de la investigación acción fueron las siguientes:

\section{a) Diagnóstico}

Tras un mes realizando observación participante, se aplicó una prueba diagnóstica con el motivo de conocer la concepción de los estudiantes respecto a la asignatura de Ciencias Sociales. Partiendo de los resultados de esta prueba y de la realización de un grupo focal con los estudiantes se concluyó que la problemática atendía a las formas tradicionales de la enseñanza de la historia.

\section{b) Diseño del plan de acción}

A raíz de las informaciones resultantes del diagnóstico, se idearon medidas pertinentes, direccionadas hacia la raíz de la problemática, para generar un cambio positivo y significativo hacia el aprendizaje de la historia. También fueron consideradas las ideas y preferencias del grupo, concibiendo así un plan con el que cada participante pudiera sentirse con interés para involucrarse.

\section{c) Implementación del plan de acción}

En esta fase, se realizaron 12 talleres. El contenido abordado fue "Historia del encuentro entre culturas en América y la colonización de la isla de Santo Domingo, siglo Xv", y se trabajó a través de actividades lúdicas para el aprendizaje significativo. Todos los talleres duraron una hora, tuvieron lugar en un aula del recinto escolar, facilitada por el equipo de gestión del propio centro educativo, y se impartieron fuera del horario de clases. A lo largo del proceso, que abarcó 12 semanas, el estudiantado fue redactando un diario reflexivo sobre sus aprendizajes y experiencias en cada uno de los talleres.

\section{d) Reflexión o evaluación}

En esta etapa se revisaron los objetivos establecidos en el desarrollo del plan de acción y se analizaron, a través de la reflexión colectiva, si fueron alcanzados y/o respondidos. Cada encuentro finalizó con una dinámica en la que los estudiantes expresaron cómo 
se sintieron respecto al taller. Las actividades realizadas en los talleres sirvieron al mismo tiempo como actividades de evaluación formativa. Como evaluación final, redactaron un ensayo y jugaron respondiendo las preguntas de un quiz virtual.

\section{Técnicas de recolección de información}

Las técnicas de recolección de datos fueron: observación participante, entrevista semiestructurada, grupos focales y de discusión y dos pruebas diagnósticas, una de las cuales también sirvió como prueba final.

En la fase de diagnóstico, se utilizó la observación participante para comprender la forma en que los participantes del proceso educativo se relacionan, las actividades tanto individuales como grupales que se realizan en aula y los recursos con los que cuentan. Esta técnica de observación se realizó por 12 horas semanales, durante cuatro semanas. También se utilizaron la entrevista semiestructurada y los grupos focales para comprender el marco conceptual que determina la didáctica de los docentes y la participación que mantienen los estudiantes. Se trabajó con un guion de 12 preguntas, y se alternaron preguntas estructuradas con otras que surgieron de manera espontánea.

La prueba diagnóstica se utilizó tanto en la fase inicial de la investigación como en la fase de evaluación, para así comparar el progreso de los estudiantes respecto a la construcción de conocimientos (Orozco, 2006). La prueba se elaboró partiendo de la decisión consensuada por los participantes de elegir el Encuentro de Culturas del siglo xv y la colonización de la isla de Santo Domingo como tema del proyecto, siendo estos contenidos que trabajaron en el curso anterior, y evaluaba las competencias específicas dictadas por el Diseño Curricular del primer grado de secundaria para este eje de contenidos: ubicación en el tiempo y el espacio, utilización critica de las fuentes de información e interacción sociocultural y construcción ciudadana (MINERD, 2016), a través de los indicadores de logro construidos con los docentes que acompañaron el proceso. Contó con 30 ítems, distri- buidos en apartados de verdadero y falso (5), selección múltiple (10), preguntas y respuestas (10) y ubicación en gráficas (5).

La recolección de la información y sus análisis ocurrieron de manera simultánea, estructurando e interpretando los datos en torno al planteamiento del problema atribuyéndoles significado y relacionándolos con teorías sobre la enseñanza de la historia.

\section{Resultados}

\section{Diagnóstico}

La información recogida y analizada en esta fase inicial permitió la descripción del fenómeno, agrupándola en cuatro categorías:

- Concepción de estrategias de enseñanza-aprendizaje de los docentes

Esta categoría inicial da a conocer el concepto personal de estrategias de enseńanza-aprendizaje que poseen los docentes desde su rol de guía del proceso educativo. En este caso, los docentes presentan ideas concretas, demostrando dominio de la parte pedagógica, y reconociendo la utilidad de las estrategias para la construcción de saberes y el desarrollo de capacidades.

"Las estrategias de aprendizaje son diversos modos, distintos caminos para alcanzar un aprendizaje significativo, que el maestro tomando en cuenta las necesidades de sus estudiantes diseña para que el proceso de enseñanza sea dinámico”. (Docente 1)

"Defino las estrategias de enseñanza como las actividades que conducen al aprendizaje. Son muy diversas porque los aprendizajes se dan de distintas formas. Para seleccionar las estrategias de enseñanza los docentes debemos tomar en cuenta los objetivos de aprendizaje y las competencias que queremos desarrollar en los estudiantes, no se deben escoger al azar ni usar las mismas siempre”. (Docente 2)

Esta descripción se relaciona con los planteamientos del MINERD (2016), que expresa en las Bases de la Revisión y Actualización Curricular que 
los educadores tienen la responsabilidad de guiar los procesos de enseñanza-aprendizaje hacia situaciones en las que el estudiantado aplique sus conocimientos, destrezas, actitudes y valores en distintos contextos, para así promover el desarrollo de las competencias. Además, los maestros tienen el compromiso de ofrecer acompañamiento y retroalimentación, así como de brindar al estudiante estrategias que le ayuden a dar sentido al mundo que los rodea a través de la transformación, construcción y reelaboración del conocimiento. En ese mismo orden, Camilloni, Cols, Basabe y Feeney (2007), plantean que es indispensable para el docente poner atención en la manera más conveniente para que los alumnos también trabajen en aquellos temas que son tratados en clase.

\section{- Concepción de Historia como asignatura}

Esta categoría explora el significado que tanto estudiantes como docentes han construido respecto a la asignatura Historia, resaltando un contraste en la visión del estudiantado y del profesorado en cuanto a la materia. Los estudiantes hablan de la historia como una herramienta para conocer el pasado, mientras que los docentes hacen referencia a la multiplicidad de competencias que se pueden desarrollar a través del estudio de ese pasado.

"Esta asignatura permite el desarrollo del pensamiento crítico, el pensamiento sistemático. Los estudiantes desarrollan la competencia cívica, la identidad cultural, y, sobre todo, la competencia histórica, con la que comprenden todo lo relativo a tiempo, espacio, hecho, causa, consecuencia”. (Docente 1)

"Esta asignatura es indispensable para que los estudiantes tengan una educación integral. La enseñanza de la historia promueve el desarrollo de la identidad local, nacional y universal de los individuos. Además de permitirles comprender los eventos del pasado, les permite comprender los hechos relevantes del presente en los aspectos políticos, sociales, culturales y económicos". (Docente 2)

Las opiniones de estos participantes guardan cierta concordancia con lo establecido por el MINERD (2016), el cual establece que los estudiantes, a través del aprendizaje de la historia, deben ubicarse en el tiempo y el espacio, y analizar cambios ocurridos en nuestro mundo. Por otra parte, muestra una concepción limitada respecto a su concepción de los fines educativos del aprendizaje de historia, a través de la cual también se comprenden hechos a partir de su contexto; se reflexiona sobre los avances y retrocesos de las naciones; se discute sobre las diferencias existentes entre países y se respeta el medio ambiente a través de acciones y posiciones críticas (MINERD, 2016).

"En esta clase aprendemos sobre hechos del pasado. Es importante porque lo que aprendemos le da sentido a las cosas". (Estudiante 1)

"Historia es conocimiento. Nos permite ver cosas que ya pasaron pero que se relacionan con el presente y nos permite tomar mejores decisiones". (Estudiante 2)

"Pienso que es importante sin embargo dan ciertas cosas que no tienen tanta relevancia en la actualidad, o por lo menos así los profesores lo hacen parecer". (Estudiante 4)

"Para mí es una asignatura muy importante porque es bueno que aprendamos sobre lo que ha pasado a lo largo de los años con el mundo para entender el presente". (Estudiante 5)

"Es la materia en la que aprendemos sobre todo lo que ha sucedido y ha sido importante en el mundo". (Estudiante 8)

"Historia es el conjunto de momentos y personajes que han marcado un antes y un después a lo largo del tiempo". (Estudiante 9)

En las ideas expresadas por estos estudiantes refleja cómo la enseñanza de la Historia se ha limitado a la búsqueda de una justificación de las acciones culturales, sociales y políticas del presente a través de la comprensión del pasado, lo que afecta significativamente la importancia que tiene para los actores del proceso educativo esta asignatura, al ignorar los demás aspectos relevantes de esta ciencia y restringiendo su narrativa a un producto terminado, cuando esta debe ser considerada como un proceso (Prats y Santacana, 2001). 
- Estrategias de enseñanza-aprendizaje de la historia

Esta categoría comprende las distintas maneras de construir el conocimiento y/o desarrollar competencias, que guía el docente en el aprendizaje de la historia y hace referencia a cómo se relaciona la motivación del estudiantado con las estrategias de enseñanza-aprendizaje contempladas.

"En los últimos años he planificado la enseñanza de los contenidos de Geografía primero que los de Historia, y lamentablemente solo me ha quedado el final del año escolar para agotar estos contenidos, $y$ por eso no he podido hacer uso de estrategias dinámicas y más elaboradas. Me gustaría utilizar videos y películas, que los estudiantes elaboraran comics. Hacer lo posible porque conmigo cambiara su actitud de apatía frente a la historia”. (Docente 1)

"El currículo educativo cada año viene sobrecargado de contenidos para los que no hay suficiente tiempo lo que representa una dificultad para nosotros. Intentamos dar todos los contenidos para que los estudiantes aprueben el curso bien preparados, pero en el fondo sé que muchas cosas se tocan muy superficialmente. Personalmente hago un esfuerzo porque puedan aprender la mayor cantidad de conceptos a través de cuestionarios y resúmenes, aunque sé que esas no son sus estrategias preferidas, pero al final es por su bien, porque van a presentarse ante pruebas para entrar a la universidad. También deben ejercitar la memorización, esta materia la requiere en gran medida”. (Docente 2)

A partir de esta expresión del docente se destacan algunas de las dificultades que señalan Reyes et al. (2009): 1) los contenidos son fraccionados, no se articulan. En este caso, si el año escolar no estuviera dividido para Geografía e Historia, los docentes serían capaces de aprovechar el tiempo de forma significativa para el aprendizaje de los estudiantes. 2) A la parte procedimental y actitudinal se le quita importancia, priorizando lo conceptual. A partir de esto, se prima el aprendizaje mecánico o memorístico en detrimento del aprendizaje significativo, para el cual se necesita mayor esfuerzo y tiempo por parte del educador.
"Siempre es lo mismo, nos ponen a hacer resúmenes, exposiciones y luego exámenes”. (Estudiante 10)

"Creo que todas las estrategias son centradas en memorización”. (Estudiante 2)

"Quisiera que hiciéramos debates y círculos de discusión para opinar sobre nuestros puntos de vista, y que no solo tuviéramos que llevar cuestionarios de 20 preguntas y hacer exposiciones tratando de repetir lo que dice el libro". (Estudiante 12)

"Casi siempre trabajamos con esquemas, mapas conceptuales. Las exposiciones nunca faltan”. (Estudiante 4)

"Pienso que las clases fueran mejor y más motivadoras si no tuviéramos que memorizar tantas fechas y personajes." (Estudiante 5)

"La mayor parte del tiempo tenemos una exposición, hacemos un resumen o un cuestionario y luego nos dan un examen." (Estudiante 9)

"Nos asignan lecturas largas para resúmenes, mapas conceptuales, esquemas y tomamos pruebines. Me gustan los esquemas." (Estudiante 14)

"Casi siempre es lo mismo, quisiera dramatizar historias, revivirlas en el presente para entender por qué son y fueron importantes, no solo exponer fechas y biografías." (Estudiante 15)

Estas citas afirman exactamente lo que desde el diseño curricular dominicano se pretende evitar. Generalmente, las secuencias didácticas más efectivas son las que combinan varias estrategias de manera pertinente y prudente para el logro de las competencias contempladas en el currículo (MINERD, 2016). Algunas de las estrategias sugeridas son el debate, técnica de estudios de casos, actividades de inserción en el entorno y dramatización, por citar algunos ejemplos, pues permiten una mayor participación del estudiantado en la construcción de su conocimiento.

- Obstáculos en el aprendizaje de la historia

Esta categoría describe las dificultades que se presentan en el proceso de enseñanza-aprendizaje de la historia, desde los recursos que se utilizan hasta la actitud de los estudiantes frente a los contenidos. 
"Es muy raro que los profesores no usen nuestro libro de texto como la fuente principal de información.” (Estudiante 1)

"Quisiera que los profesores utilizarán otros recursos, no solo el libro, para ayudarnos a aprender, como la música y el juego, cosas más dinámicas que nos motiven porque de por sí los temas son aburridos." (Estudiante 3)

"Si las clases fueran más interactivas aprendiéramos más. Suelen ser aburridas." (Estudiante 4)

"Los profesores deberían utilizar más recursos, escuchar y escuchar cansa." (Estudiante 7)

"Nos gustaría visitar lugares históricos como museos y sitios donde sucedieron cosas importantes." (Estudiante 16)

"Utilizamos el libro en todas las clases, a veces vemos un documental." (Estudiante 11).

En estos comentarios se destaca, tal y como puntualizan Reyes et al. (2009), que el uso excesivo de determinados métodos o recursos desmotiva al estudiantado. El abuso del libro de texto como principal material didáctico es una práctica recurrente, pero el desarrollo cognoscitivo y psicomotor requieren que el estudiante interactúe con diversos medios y recursos tal como lo establece el Diseño Curricular del Nivel Secundario. Este establece que, aparte de libros, es necesario el uso de textos diversos, de tablas e histogramas estadísticos, documentos históricos, almanaques, diccionarios, materiales cartográficos, atlas, revistas, tratados antiguos y guías de ciudades (MINERD, 2016).

"Uno se embotella la información, la escribe en el examen y al otro día no recuerda lo que estudió." (Estudiante 4)

"Es una de mis materias favoritas no soy buena memorizando información y siento que no aprendo." (Estudiante 6)

"Es una materia aburrida, repetimos lo que está en el libro sin cuestionarlo ni discutirlo la mayoría de las veces porque no hay suficiente tiempo y hay que avanzar los temas." (Estudiante 10)

"Generalmente los estudiantes presentan animadversión frente a la historia. Pero yo comprendo que eso viene del hecho de que tradicionalmente solo se ha trabajado con memorización, repetición de hechos. No se les pide que interpreten los hechos y transformen realidades. Desde que se les habla de Historia creen que se les asignará un cuestionario de 30 preguntas y que repetirán información como papagayos." (Docente 1)

Aquí vemos dos espejos de una realidad. Evidentemente, para aprender es necesaria la memorización, pero cuando solo se memoriza no se está cumpliendo con lo necesario para aprender significativamente una disciplina como la historia, la cual requiere del desarrollo de valores, actitudes y pensamiento crítico (Reyes et al., 2009).

En esta fase se impartió la prueba diagnóstica elaborada por los investigadores junto a los docentes, que tuvo como objetivo principal conocer el estado inicial de los estudiantes respecto al conocimiento sobre los contenidos elegidos para trabajar en la fase de intervención, y así adaptar los objetivos del proceso de aprendizaje a sus necesidades. Los principales resultados fueron que el estudiantado reconocía fechas y personajes principales, pero no eran capaces de señalar antecedentes, causas ni consecuencias de los hechos históricos, ni los concebían como procesos que se relacionan con la realidad actual de todas las partes afectadas. De igual modo, no ubicaban el espacio geográfico en el que se desarrollaron los hechos ni establecían diferencias económicas, políticas y culturales entre las sociedades taína y española.

\section{Diseño e implementación del plan de acción}

Teniendo en cuenta las necesidades de aprendizaje plasmadas en el diagnóstico de esta investigación, se diseñó una propuesta de acción con el objetivo de incidir de forma positiva en la experiencia de aprendizaje de los estudiantes y la práctica de sus docentes. Se presentó Playstory, creado por los investigadores, como un programa de acción que buscó responder a las necesidades relativas al proceso de enseñanza-aprendizaje de la historia, ofreciendo a los estudiantes una visión diferente de esta área del conocimiento a través de una enseñanza lúdica que permita una mayor comprensión del mundo en que 
viven mediante el desarrollo del pensamiento crítico. Playstory abarcó una unidad de contenidos sobre la Historia Colonial de la Isla de Santo Domingo (desde el encuentro entre culturas hasta las devastaciones de Osorio en 1606). Esta unidad de contenidos fue seleccionada atendiendo a los intereses de los estudiantes, quienes expresaron que era un tema tratado en grados anteriores, pero no lo dominaban lo suficiente.

Atendiendo a la concepción de los estudiantes en cuanto a las estrategias de enseñanza-aprendizaje utilizadas por los docentes respecto a la enseñanza de la historia, se implementaron, como parte del plan de acción, estrategias de enseñanza-aprendizaje lúdicas, combinando la construcción de conocimientos junto con la diversión y el placer. Estas estrategias son promovidas por autores de la Neuroeducación, como Jesús Guillén (2017), quien establece que estas actividades son pertinentes para el proceso educativo, gracias a los neutransmisores segregados en el cerebro que se encuentran relacionados con la motivación y la satisfacción: dopamina, oxitocina, endorfinas y serotonina. De igual forma se incluyeron estrategias propuestas por el Diseño Curricular (MINERD,
2016), que apuntan al desarrollo de las competencias específicas y fundamentales, como el debate y el sociodrama. Asimismo, se utilizó un conjunto más amplio de recursos educativos, como materiales cartográficos, documentos históricos, vídeos informativos.

Durante el desarrollo de los talleres los docentes y las docentes recibían reportes semanales del trabajo realizado en cada taller. El estudiantado mostró un alto nivel de motivación durante todo el proceso, y mantuvo una asistencia constante a pesar de que los talleres se realizaron fuera del horario de clases como una actividad extracurricular no obligatoria.

\section{Evaluación}

Para evaluar los aprendizajes fueron utilizadas tanto actividades como instrumentos. Así como establece el MINERD (2016), cualquier actividad planificada para evaluar es al mismo tiempo una actividad de aprendizaje. En la siguiente tabla, presentamos las técnicas que utilizamos para realizar la evaluación de los aprendizajes en cada taller.

Tabla 1. Contenidos y técnicas de evaluación

\begin{tabular}{|c|c|c|c|}
\hline Tema & Objetivo & Actividad Central & Técnicas de Evaluación \\
\hline $\begin{array}{l}\text { Dos Mundos diferentes: ¿Cómo } \\
\text { eran las } \\
\text { cosas antes del encuentro? }\end{array}$ & $\begin{array}{l}\text { Distinguir las características } \\
\text { de las sociedades y culturas } \\
\text { taína y española del siglo XV. }\end{array}$ & $\begin{array}{l}\text { Elaboración de } \\
\text { mapamundi del siglo XV, } \\
\text { Debate “¿Cuál sociedad } \\
\text { era más avanzada al } \\
\text { momento del encuentro? } \\
\text { Taína vs espańola”. }\end{array}$ & $\begin{array}{l}\text { Lista de cotejo sobre los } \\
\text { elementos representados en el } \\
\text { mapa. } \\
\text { Debate para evaluar la } \\
\text { valoración de las sociedades en } \\
\text { relación con cómo responden a } \\
\text { sus necesidades. }\end{array}$ \\
\hline $\begin{array}{l}\text { El plan de Colón: ¿Qué } \\
\text { problemática impulsó el primer } \\
\text { viaje de Colón y, finalmente, el } \\
\text { encuentro? }\end{array}$ & $\begin{array}{l}\text { Conocer el contexto } \\
\text { sociohistórico y los intereses } \\
\text { políticos detrás del viaje de } \\
\text { Colón. }\end{array}$ & $\begin{array}{l}\text { Juego de pistas sobre el } \\
\text { contexto sociohistórico } \\
\text { tras el viaje de Colón. } \\
\text { Elaboración de cartel } \\
\text { con cronología sobre los } \\
\text { antecedentes de la llegada } \\
\text { de Colón. }\end{array}$ & $\begin{array}{l}\text { Rúbrica para evaluar el producto } \\
\text { y su presentación. }\end{array}$ \\
\hline
\end{tabular}




$\begin{array}{lll}\text { Tema } & \text { Objetivo } & \text { Actividad Central }\end{array}$

Recuperación de los saberes

obtenidos en las clases anteriores.

obtenidos en las clases anteriores.

El encuentro: la llegada y los primeros contactos entre aborígenes y europeos.

La represión y resistencia en el proceso de colonización: ¿De qué forma controlaron los españoles a los indígenas?

El sistema de encomiendas como mecanismo de dominación: ¿̨Por qué no funcionó el sistema de encomiendas?

El intercambio de flora y fauna entre las dos sociedades: su influencia en nuestra dieta actual.

El contrabando: corso-piratería: los verdaderos piratas del Caribe.

Devastaciones de Osorio: su influencia en la creación de una segunda colonia en la isla de Santo Domingo.
Fijar los conocimientos obtenidos en las clases anteriores.

Repensar el llamado "Descubrimiento de

América”, comprendiéndolo como un encuentro entre culturas.

Conocer los mecanismos de control utilizados en el sistema colonial; aculturación, transculturación.

Reflexionar sobre la causa el fracaso del sistema de encomiendas; la extinción indígena.

Valorar el impacto socioeconómico y cultural que produjo el intercambio colombino.

Comprender los conceptos de corso y piratería. Reconstruir la figura del pirata: más fiel a la realidad y menos a la televisión.

Identificar y comprender las causas y consecuencias de las Devastaciones de Osorio, así como su impacto en la formación de nuevas ciudades,
Quiz con Kahoot. Quiz con Kahoot.

Sociodrama representando la perspectiva aborigen de Redacción de un diario reflexivo. la llegada de los españoles.

Creación de una canción sobre los personajes Guacanagarix y Caonabo.

Lista de cotejo.

Interpretación de un acuerdo de encomiendas y caracterización de un encomendado.

Mapa conceptual sobre la encomienda.

Juego de recolección de alimentos que fueron parte del intercambio

Quiz Kahoot. colombino.

Caracterización del personaje: "Soy un pirata".

Lista de cotejos.

Juego de pistas para recrear mapa a través de un rompecabezas de la división de la isla en ese momento de la historia.
Lista de cotejos para la presentación del producto.

Diario reflexivo.

Fuente: elaboración propia.

A partir de las técnicas de evaluación que fueron utilizadas, la observación de los docentes y los resultados de la prueba diagnóstica/final, el rendimiento de los estudiantes demuestra que fueron alcanzados la mayoría de los indicadores de logro. 
Tabla 2. Indicadores de logro - Playstory

\begin{tabular}{|c|c|c|}
\hline Indicador de logro & Alcanzado & No alcanzado \\
\hline $\begin{array}{l}\text { Establece diferencias entre la sociedad espańola y la sociedad taína a partir de } \\
\text { sus características sociales, económicas y culturales en el siglo xv. }\end{array}$ & $\mathrm{X}$ & \\
\hline $\begin{array}{l}\text { Ubica en un mapamundi el territorio ocupado por la sociedad española y la } \\
\text { sociedad taína en el siglo xv. }\end{array}$ & $\mathrm{X}$ & \\
\hline Organiza cronológicamente los antecedentes del primer viaje de Colón. & & $\mathrm{X}$ \\
\hline Traza en un mapamundi la ruta del primer viaje de Colón. & $\mathrm{X}$ & \\
\hline Opina sobre la reacción de los aborígenes ante la llegada de Colón. & $\mathrm{X}$ & \\
\hline $\begin{array}{l}\text { Opina sobre las impresiones de Cristóbal Colón al llegar al Caribe a partir de } \\
\text { su diario. }\end{array}$ & $\mathrm{X}$ & \\
\hline Ubica en un mapa del Caribe la primera isla conocida por los españoles. & $\mathrm{X}$ & \\
\hline Identifica las causas de los primeros enfrentamientos entre taínos y españoles. & & $\mathrm{X}$ \\
\hline Debate respecto a las decisiones de Guacanagaríx y Caonabo. & $\mathrm{X}$ & \\
\hline $\begin{array}{l}\text { Valora el sistema de encomiendas como un mecanismo de dominación } \\
\text { cultural y social. }\end{array}$ & $\mathrm{X}$ & \\
\hline Identifica las causas del fracaso del sistema de encomiendas. & $\mathrm{X}$ & \\
\hline $\begin{array}{l}\text { Identifica los recursos y productos originarios de América y los importados } \\
\text { desde África, Asia y Europa. }\end{array}$ & $\mathrm{X}$ & \\
\hline Valora la relevancia del intercambio colombino en la actualidad. & $\mathrm{X}$ & \\
\hline Diferencia entre piratas y corsarios. & $\mathrm{X}$ & \\
\hline Conoce las hazañas de piratas populares en la región del Caribe. & $\mathrm{X}$ & \\
\hline Identifica causas y consecuencias de las vastaciones de Osorio. & $\mathrm{X}$ & \\
\hline $\begin{array}{l}\text { Ubica en un mapa de la Isla de Santo Domingo las ciudades devastadas y } \\
\text { fundadas luego de la devastación. }\end{array}$ & $\mathrm{X}$ & \\
\hline
\end{tabular}

Fuente: elaboración propia.

Cabe destacar que los estudiantes reconocieron durante todo el proceso de aprendizaje la importancia del trabajo colaborativo, así como la reflexión crítica colectiva. El Diseño Curricular orienta que las actividades realizadas en clase deben estar orientadas al desarrollo de habilidades de convivencia, al intercambio colectivo de aprendizajes y al alcance de metas en conjunto (MINERD, 2016). En sus reflexiones, los participantes expresan lo siguiente:
"Cada clase de Playstory fue una aventura nueva en la que aprendimos trabajando en equipos. Trabajar junto a mi compañera me permitió tener un mejor desempeńo y me ayudó a desarrollar más mis ideas”. (Estudiante 2)

"Además de expandir mi conocimiento en historia, puedo decir que crecí como persona gracias a los retos que tuvimos que cumplir en grupos." (Estudiante 4) 
Uno de los tantos beneficios de la enseñanza de historia es que su aprendizaje permite la comprensión del presente, ofreciendo una perspectiva rica en relevancia a través del análisis del pasado, estudiando la causa y consecuencia de los sucesos, fomentando la construcción de sistemas de similitudes y diferencias, examinando los cambios de las sociedades a través del tiempo e interpretando la dificultad de estas variaciones (Prats y Santacana, 2001). Esto es reconocido por los estudiantes al expresar que:

"A partir del aprendizaje de la historia de nuestra isla pude comprender más aspectos de nuestra cultura y cómo este proceso determinó ciertas cuestiones de la sociedad en que vivimos". (Estudiante 1)

En virtud de que el uso del libro de texto como único recurso de aprendizaje en historia significa una dificultad, en Playstory se implementó una variedad de recursos que facilitaron la construcción de saberes, desde el mismo campus del colegio, hasta películas y documentos históricos de la época. Estos recursos son sugeridos en el Diseño Curricular (MINERD, 2016), pues estimulan el desarrollo del pensamiento crítico, lógico y creativo, mejoran la experiencia sensorial y desarrollan la capacidad de conceptualización desde lo concreto.

"Los recursos que utilizamos en la clase nos ayudaron a comprender fácilmente los temas. Leer el diario de Cristóbal Colón nos proporcionó informaciones que no siempre aparecen en el libro y que nos permitieron comprender su punto de vista y compararlo con otros personajes que escribieron sobre los taínos." (Estudiante 13)

"Vimos las películas famosas hechas sobre los piratas y pudimos comparar la visión común que se tiene sobre estos personajes de la historia del Caribe y Europa, con quienes en realidad fueron." (Estudiante 14)

"En una clase utilizamos elementos de todo el espacio del colegio como recursos y fue muy interesante." (Estudiante 3)

Como mencionan los estudiantes, el uso de estos recursos los colocó en un contacto más cercano a los contenidos, amplió su comprensión sobre los mismos y fomentó el desarrollo de una perspectiva más crítica.
Las estrategias de enseñanza-aprendizaje lúdicas estuvieron presentes durante el desarrollo de la unidad de contenidos, alternándose con las estrategias basadas en el aprendizaje significativo sugeridas por el currículo del país. Estas actividades lúdicas combinan el placer y la diversión con el aprendizaje, mientras que las estrategias sugeridas por el MINERD (2016) están orientadas al desarrollo del aprendizaje significativo, la actividad constructiva, la reflexión y la colaboración.

"Normalmente para aprender historia o ciencias sociales utilizamos la memorización y esto nos hace más difícil el aprender los temas. Tomando esta clase me he dado cuenta de que podemos aprender no solo memorizando, sino interactuando, trabajando en grupos y divirtiéndonos, y aparte de que es divertido, nos ayuda a aprender de verdad." (Estudiante 1)

"Con este método de enseñanza aprendí temas que no había logrado aprender en otros cursos, como la conquista de la isla y las devastaciones de Osorio. Me gustó mucho el ambiente y cómo nos sentíamos libres de expresar nuestra opinión sin miedo a equivocarnos." (Estudiante 9)

"En esta clase descubrí que la historia no es aburrida si se cambia la forma de enseñanza por una más dinámica, las actividades despertaron mi curiosidad por aprender más." (Estudiante 15)

"Decidí inscribirme en Playstory con las expectativas de que las clases serían aburridas porque pensaba que la historia siempre era aburrida, pero gracias a la forma en que nos enseñaron pude aprender muchas cosas nuevas y comprender cosas que había visto antes pero que no había entendido realmente." (Estudiante 14)

"Siento que además de aprender historia también crecí como persona porque trabajamos dándole importancia a los valores. Me sentí muy cómoda en el ambiente y libre de expresarme y participar sin miedo a equivocarme y a ser juzgada gracias a los juegos." (Estudiante 5)

"Desde el primer día me di cuenta de que no solo estábamos allí para aprender sino para hacerlo divirtiéndonos y eso me permitió tener una actitud positiva en cada clase y aprender más.” Estudiante 16 
En estos comentarios se percibe cómo el uso de estas estrategias guarda una relación directa con la comunicación y el aprendizaje en el aula. Así como expresan Córdoba et al. (2017), las actividades lúdicas estimulan el desarrollo de aspectos sociales, la capacidad intelectual, la emocional y la comunicativa. En ese orden, los estudiantes expresan rechazo hacia la memorización mecánica como estrategia y conformidad respecto al aprendizaje divorciado de lo aburrido y cercano a lo interactivo y divertido, argumentando que a través de esto construyen aprendizajes significativos ("de verdad"). Esto se resume en que existe mayor motivación, y como sostiene Guillén (2017), durante el juego se segrega la dopamina, entre otros neurotransmisores, la cual mantiene un estado motivacional óptimo en el individuo.

\section{Conclusiones}

El presente trabajo relata una experiencia de investigación-acción sobre estrategias lúdicas para la enseñanza de la historia en el nivel secundario. Entre los principales hallazgos podemos resaltar que las estrategias de enseñanza tradicionales basadas en la memorización y repetición de contenidos inciden negativamente en la concepción que desarrollan los estudiantes sobre la asignatura, limitando su importancia al conocimiento del pasado, y dejando de lado el conjunto de competencias que pueden desarrollar a través de su aprendizaje. Asimismo, tienden a desarrollar cierto sentido de apatía ante los contenidos y su rendimiento se caracteriza por bajos niveles de motivación perpetuados por la falta de innovación en el uso de recursos educativos. Una vez realizada la intervención, los resultados del proceso educativo demuestran que los estudiantes aprenden historia significativamente cuando se fomenta su participación activa, utilizando estrategias estrechamente relacionadas al aumento de la motivación, acompañando el proceso de aprendizaje con recursos que sirvan de puente entre los saberes previos y el conocimiento nuevo, y con un abordaje que tome en cuenta todos los beneficios de la enseñanza de Historia, y no los restrinja al mero conocimiento del pasado.
El sistema educativo dominicano aspira a la formación de individuos que piensen de forma crítica, reflexiva y analítica, que se reconozcan a sí mismos como parte de la comunidad nacional y global, con suficiente conciencia histórica como para asumir el compromiso de contribuir a la construcción de una sociedad caracterizada por el ejercicio de la justicia, la responsabilidad y la solidaridad (MINERD, 2016). Para que esto ocurra es necesario la concreción del currículo de Ciencias Sociales de forma satisfactoria. El análisis presentado indica que la enseñanza de la historia está caracterizada por prácticas deficientes que han impulsado la narrativa de que es aburrida e innecesaria en el imaginario de muchos estudiantes, alejando la posibilidad de la construcción de un aprendizaje con sentido para el estudiantado.

Un sistema educativo que concibe el aprendizaje como un proceso de construcción, no de transmisión de conocimientos, y que está diseñado para el desarrollo del saber, saber hacer y saber ser propone diversos modos para la enseńanza de los contenidos que son parte del desarrollo integral de los estudiantes. Aun así, y a pesar de poseer el dominio conceptual necesario para implementar estos nuevos modos de enseñar, se aprecia cómo muchos docentes han permanecido utilizando los métodos clásicos en la enseñanza de la historia, cerrando las puertas a las múltiples competencias que podrían ser desarrolladas a través de novedosas estrategias. Sumado a esto es necesario destacar la recurrencia del libro de texto como principal recurso educativo, lo cual contribuye a la monotonía en los planes y desarrollo de las clases, limitando el interés por el aprendizaje. El uso de diferentes recursos, sin restarle importancia a los libros de texto, supone mayores niveles de motivación y participación de los estudiantes, pues ofrecen la oportunidad de atender a una mayor variedad de estilos de aprendizaje.

El estudio también muestra evidencia de que la animadversión de los estudiantes ante la asignatura de la historia es modificable, pues depende significativamente de la forma en que esta sea enseñada. Es importante reflexionar respecto a las clases de Historia basadas, principalmente, en la exposición 
del docente y la memorización de fechas o hazañas de personajes históricos. Desde la innovación en las prácticas educativas para la calidad del aprendizaje se propone integrar actividades que involucren a los estudiantes en su rol activo dentro del proceso de construcción de conocimientos. Cuando el docente integra actividades en las que el estudiante asume un rol activo en su aprendizaje, estos demuestran la capacidad de ser creativos y reflexivos, contrario a lo que sucede cuando no se perciben cómo protagonistas de sus aprendizajes, sino como receptores de información, que serán luego evaluados por su habilidad para repetirla.

Es responsabilidad del cuerpo docente asumir el compromiso de enseñar la Historia como procesos de construcción de conocimiento, para una mejor comprensión del mundo actual y para el fomento de una convivencia democrática y sana (MINERD, 2016), incorporando estrategias de enseńanza-aprendizaje apropiadas, desde un enfoque constructivista y por competencias, y con el auxilio de recursos que propicien el debate y el diálogo, para la formación de individuos transformadores de la sociedad.

Consideramos que es pertinente enseñar al estudiantado que la historia es construida desde diferentes escuelas historiográficas y además por autores con diversas ideologías. Asimismo, hacer énfasis en que, al igual que toda información, la narración de los hechos puede ser manipulada para ceñirse a los intereses de las esferas del poder. Por lo tanto, la utilización del libro de texto como único recurso es evidentemente una limitante para el análisis histórico. Es preciso también hacer que los jóvenes consideren que las generalizaciones son solamente eso, conclusiones a partir de fenómenos determinados observados en una mayoría de casos. Es decir, que es un error totalizar cuando se generaliza. Todo esto es parte de una competencia transversal: selección, uso y análisis de la información. Asimismo, es importante conectar los hechos históricos de manera secuencial y no mostrarlos como sucesos aislados unos de otros. Lo apropiado es basarse en el modelo explicativo de causa y efecto, pero no solo en relación con una unidad o tema. Es decir, que la práctica ideal no se basa solo en identificar las causas y consecuencias de un hecho histórico, sino qué implicaciones tuvo este movimiento para la posteridad no inmediata y qué implicaciones conserva con relación al presente. Por otro lado, se considera oportuno también conectar los hechos, aunque no tengan alguna relación más que el momento en el que ocurrieron. Dicha práctica favorece la competencia "ubicación en el espacio-tiempo". Es necesario enseñar prestando más atención al componente actitudinal, sin dejar de lado lo conceptual y lo procedimental. Con este enfoque se promueve un mayor acercamiento entre el estudiante y el contexto histórico a través de múltiples emociones como la empatía.

\section{Referencias}

Abero, L., Berardi, L., Capocasale, A., García, S. y Rojas, R. (2015). Investigación educativa: abriendo las puertas al conocimiento. Contexto S.R.L. Recuperado de https://bit.ly/2ynK7hI

Araujo, M., Gómez, N., Fonseca, F. y Molano, W. (2013). Estrategia de enseñanza-aprendizaje basada en la lúdica en tercero de primaria. Revista Infancias Imágenes. 12(1), 89-98. Recuperado de https://bit.ly/2T3Xprrs

Benítez, G. (2010). Las estrategias de aprendizaje a través del componente lúdico. Revista de Didáctica Español Lengua Extranjera, (11), 1-68. Recuperado de https://bit.ly/32HWAbn

Camilloni, A., Cols, E., Basabe, L. y Feeney, S. (2007). El saber didáctico. Buenos Aires: Ediciones Paidós. Recuperado de https://bit. ly/2RNtG5g

Casal, S. (2011). Aprender Historia en la escuela secundaria: el caso de Morelia, Michoacán (México). Revista mexicana de investigación educativa, 16(48), 73-105. Recuperado de https://bit.ly/2VzSdvC

Cepeda, J. M. (2013). Estrategias de Enseñanza para el Aprendizaje por Competencias. Jesús Martín Cepeda Dovala. Recuperado de https://bit. ly/3ajw3U2 
Córdoba, E. F., Lara, F. y García, A. (2017). El juego como estrategia lúdica para la educación inclusiva del buen vivir. Revista de la Facultad de Educación de Albacete, 32(1), 1-12. Recuperado de https://bit.ly/39PiPhq

Guerrero, M. A. (2016). La Investigación Cualitativa. INNOVA Research Journal, 1(2), 1-9. Recuperado de https://doi.org/10.33890/ innova.v1.n2.2016.7

Guillén, J. (2017). Gamificación desde la neuroeducación. [Entrada en un blog]. Recuperado de https://bit.ly/3cjwGPB.

Hurtado, P. A., García, M., Rivera, D. A. y Forgiony, J. O. (2018). Las estrategias de aprendizaje y la creatividad: una relación que favorece el procesamiento de la información. Revista Espacios, 39(17), 1-18. Recuperado de https://bit. ly/2JNRhyi.

Martínez, L. V. y Pérez, M. D. (2015). Gamificación: Estrategia para optimizar el proceso de aprendizaje y la adquisición de competencias en contextos universitarios. Digital Education Review, 27, 13-31. Recuperado de https://doi. org/10.1344/der.2015.27.13-31. https://bit. ly/2XHKoqi

Ministerio de Educación de la República Dominicana. (2016). Bases de la Revisión y Actualización Curricular. Santo Domingo: MINERD. Recuperado de https://bit.ly/389g1up

Ministerio de Educación de la República Dominicana. (2016). Diseño curricular nivel secundario, primer ciclo. Santo Domingo: MINERD. Recuperado de https://bit.ly/2wcvInk

Ministerio de Educación dela República Dominicana. (2016). Informe curricular de los resultados de las Pruebas Nacionales, Primera y Segunda Convocatorias. Santo Domingo: MINERD. Recuperado de https://bit.ly/2XI97e7

Ministerio de Educación de la República Dominicana. (2017). Informe curricular de los resultados de las Pruebas Nacionales, Primera y Segunda Convocatorias. Santo Domingo: MINERD. Recuperado de https://bit.ly/2ynZRS1
Ministerio de Educación de la República Dominicana (2018). Informe curricular de los resultados de las Pruebas Nacionales, Primera y Segunda Convocatorias. Santo Domingo: MINERD. Recuperado de https://bit.ly/2zbxKG3

Molina, S., Miralles, P., Deusdad, B. y Begoña, M. (2017). Enseñanza de la historia, creación de identidades y prácticas docentes. Profesorado. Revista de Currículum y Formación de Profesorado, 21(2), 331-354. Recuperado de https:// bit.ly/2V44HLC

Moreira, M. (2017). Aprendizaje significativo como un referente para la organización de la enseñanza. Archivos de Ciencias de la Educación, 11(12), 1-16. Recuperado de https://doi. org/10.24215/23468866e029

Lewin, K. (1992). La investigación-acción y los problemas de las minorías. En M. Salazar (ed.). La investigación-acción participativa: inicios y desarrollos (pp. 13-26). Madrid: Editorial Popular. Recuperado de https://bit. ly/3cWjYps

Orozco-Jutorán, M. (2006). “La evaluación diagnóstica, formativa y sumativa en la enseńanza de la traducción”, en: M.J. Varela (ed.). La evaluación en los estudios de traducción e interpretación termina cursiva. Sevilla: Bienza. Recuperado de https://bit.ly/2I3uCx7

Otzen, T. y Manterola, C. (2017). Técnicas de Muestreo sobre una Población a Estudio. International Journal of Morphology, 35(1), 227-232. Recuperado de https://dx.doi.org/10.4067/ S0717-95022017000100037

Pérez-Van-Leenden, M. (2019). La investigación acción en la práctica docente. Un análisis bibliométrico (2003-2017). Magis, Revista Internacional de Investigación en Educación, 12(24), 177-192. Recuperado de https://doi. org/10.11144/Javeriana.m12-24.ncev. https:// bit.ly/2XCm9Kr

Pluckrose, H. (1996). Enseñanza y aprendizaje de la historia. Madrid: Ediciones Morata. Recuperado de https://bit.ly/2ShqIqb 
Prats, J. C. (2007). La Historia es cada vez más necesaria para formar personas con criterio. Revista Escuela, (3.753), 22-23. Recuperado de https:// bit.ly/2Tl1qGX

Prats, J. C. y Santacana, J. (2001). Enseñar Historia: notas para una didáctica renovadora. Mérida: Junta de Extremadura. Recuperado de https:// bit.ly/2VtMtoI

Reyes, J., Jevey, A., Guerra, S., Palomo, A. y Romero, M. (2009). Estrategias de enseñanza y aprendizaje de la historia en la escuela. La Habana: Educación Cubana. Recuperado de https://bit. ly/2I1IyYA

Sáiz, J. y Fuster, C. (2014). Memorizar historia sin aprender pensamiento histórico: las PAU de Historia de España. Revista Investigación en la Escuela, 84, 47-57. Recuperado de https://bit. ly/32z9V5K
Sosa, F. y Rodríguez, C. (2010). Métodos y Técnicas de la Enseñanza de la Historia y la Geografía en el Segundo Ciclo del Nivel Medio en el Liceo Público del Distrito Educativo 08-03 de Santiago 20082009. [Tesis de Grado]. Instituto Superior de Formación Docente Salomé Ureña. Recuperado de https://bit.ly/2PCDOwA

Vallori, A., Enrique, P., Payeras, J. y Xamena, G. (2002). El aprendizaje significativo en la práctica y didáctica de la geografía: prácticas del Seminario de aprendizaje significativo. Revista educación y pedagogía, 14(34), 97-110. Recuperado de https://bit.ly/2TbVsJC 